\title{
Bio-technology of using photogenic feed additives in poultry diets (Abstract)
}

\author{
S. A. Abd EL-Latif \\ Department of Animal Production, Faculty of Agriculture, Minia University, Minia, Egypt \\ Corresponding author: profshaker71@yahoo.com
}

\begin{abstract}
The use of feed additives is usually subject to restrictive regulations. In general, they are considered as products applied by the farmer to healthy animals for a nutritional purpose on a permanent basis (i.e., during the entire production period of the respective species and category), in contrast to veterinary drugs (applied for prophylaxis and therapy of diagnosed health problems under veterinarian control for a limited time period, partially associated with a waiting period). Plant species from the families of Zingiberaceae (e.g., ginger and curcuma) and Umbelliferae (e.g., anise and coriander), as well as plants rich in flavonoids (e.g., green tea) and anthocyans (e.g., many fruits), are also described as exerting antioxidative properties. Furthermore, pepper (Piper nigrum), red pepper (Capsicum annuum L.), and chili (Capsicum frutescene) contain antioxidative components. In many of these plants, parts of the active substances are highly odorous or may taste hot or pungent, which may restrict their use for animal feeding purposes. The antioxidant property of many phytogenic compounds may be assumed to contribute to protection of feed lipids from oxidative damage, such as the antioxidants usually added to diets (e.g., $\alpha$-tocopheryl acetate or butylated hydroxytoluene). Although this aspect has not been explicitly investigated poultry feeds, there is a wide practice of successfully using essential oils, especially those from the Labiatae plant family, as natural antioxidants in human food, as well as in the feed of companion animals. The objective of this review was to evaluate the Bio-technology of using photogenic feed additives in poultry diets.
\end{abstract}

Keywords: Bio-technology, feed additives, poultry, diets 
